\title{
Unifying Multimodal Transformer for Bi-directional Image and Text Generation
}

\author{
Yupan Huang* \\ Sun Yat-sen University \\ huangyp28@mail2.sysu.edu.cn \\ Bei Liu \\ Microsoft Research Asia \\ Bei.Liu@microsoft.com
}

\author{
Hongwei Xue* \\ University of Science and Technology of China \\ gh051120@mail.ustc.edu.cn
}

\author{
Yutong $\mathrm{Lu}^{\dagger}$ \\ Sun Yat-sen University \\ luyutong@mail.sysu.edu.cn
}

\begin{abstract}
We study the joint learning of image-to-text and text-to-image generations, which are naturally bi-directional tasks. Typical existing works design two separate task-specific models for each task, which impose expensive design efforts. In this work, we propose a unified image-and-text generative framework based on a single multimodal model to jointly study the bi-directional tasks. We adopt Transformer as our unified architecture for its strong performance and task-agnostic design. Specifically, we formulate both tasks as sequence generation tasks, where we represent images and text as unified sequences of tokens, and the Transformer learns multimodal interactions to generate sequences. We further propose two-level granularity feature representations and sequence-level training to improve the Transformer-based unified framework. Experiments show that our approach significantly improves previous Transformer-based model X-LXMERT's FID from 37.0 to 29.9 (lower is better) for text-to-image generation, and improves CIDEr-D score from $100.9 \%$ to $122.6 \%$ for fine-tuned image-to-text generation on the MS-COCO dataset. Our code is available online.
\end{abstract}

\section{CCS CONCEPTS}

- Computing methodologies $\rightarrow$ Natural language generation; Image processing.

\section{KEYWORDS}

cross-modal generation; image captioning; text-to-image synthesis

\section{ACM Reference Format:}

Yupan Huang, Hongwei Xue, Bei Liu, and Yutong Lu. 2021. Unifying Multimodal Transformer for Bi-directional Image and Text Generation. In Proceedings of the 29th ACM International Conference on Multimedia (MM '21), October 20-24, 2021, Virtual Event, China. ACM, New York, NY, USA, 10 pages. https://doi.org/10.1145/3474085.3481540

\footnotetext{
*Work done during an internship at Microsoft Research Asia.

${ }^{\dagger}$ Corresponding Author.

Permission to make digital or hard copies of all or part of this work for personal or classroom use is granted without fee provided that copies are not made or distributed for profit or commercial advantage and that copies bear this notice and the full citation on the first page. Copyrights for components of this work owned by others than ACM must be honored. Abstracting with credit is permitted. To copy otherwise, or republish, to post on servers or to redistribute to lists, requires prior specific permission and/or a fee. Request permissions from permissions@acm.org.

MM '21, October 20-24, 2021, Virtual Event, China

(c) 2021 Association for Computing Machinery.

ACM ISBN 978-1-4503-8651-7/21/10 . \$ $\$ 15.00$

https://doi.org/10.1145/3474085.3481540
}

(1) Task-specific uni-directional architectures

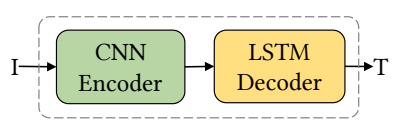

(a) I $\rightarrow$ T (e.g., BUTD)

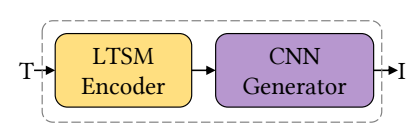

(b) $\mathrm{T} \rightarrow \mathrm{I}$ (e.g., DM-GAN)
(2) Task-agnostic bi-directional architectures

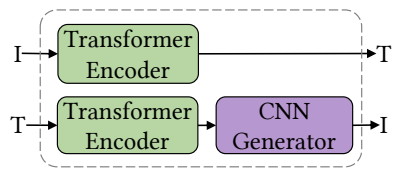

(c) I $\longrightarrow$ T (e.g., X-LXMERT-FT)

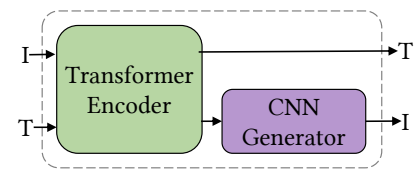

(d) $\mathrm{I} \longleftrightarrow \longrightarrow \mathrm{T}$ (Ours)
Figure 1: Comparison with existing works [2, 6, 53] on bi-directional image and text generation tasks. Our taskagnostic bi-directional architecture as show in (2) releases design efforts of task-specific architectures in (1). When comparing with other Transformer-based models in a pretrain and fine-tune fashion as shown in (c), our model unifies two tasks in a Transformer model. "X-LXMERT-FT" denotes fine-tuning X-LXEMRT [6] for two tasks. "I" and "T" denote "image" and "text" respectively.

\section{INTRODUCTION}

As a result of developing multimodal processing technology, networks, and devices, the number of existing multimodal industrial applications (especially mobile applications) is rapidly increasing. This trend has encouraged the development of techniques to support multimodal interaction in a unified system. Bi-directional image and text generation is a technique that automatically translates across real scene images and natural language descriptions. It has a broad range of industrial applications. For example, a product description-and-picture generation system is an important application for consumers to search and preview products. Specifically, consumers could easily get a text query when the system receives a picture of one or several products. Meanwhile, the users could say "show me the picture of a room with wooden furniture and purple curtains." and receive an illustrating picture by the system to preview this collocation. As these applications are equipped in mobile devices, unifying multimodal interactions in a single model will be a better choice to optimize storage utilization compared to two separate models. 
Despite the great benefit of a unified framework for image and text generation, the bi-directional generation tasks are conducted separately with task-specific architectures traditionally. As shown in Figure 1 (1), a typical image-to-text generator consists of a visual encoder (e.g., CNN) to embed visual information, and a caption decoder (e.g., LSTM) to generate captions [2]; while predominant text-to-image generators adopt Generative Adversarial Nets (GANs) [11] framework based on CNN architectures [53]. To support multimodal interaction, Huang et al. jointly trains an LSTM-based image-to-text generator and a GAN-based text-to-image generator in a framework [17]. However, task-specific architectures are still needed, which introduces expensive design efforts.

To alleviate above hassles, in this paper, we propose to unify image-to-text and text-to-image generation tasks in one framework. In this framework, we adopt Transformer-based architecture since it supports simple and task-agnostic designs, and exhibits strong performance in image or text generative models [27, 36]. We formulate both tasks as sequence generation tasks, where an image and a text are represented as sequences of tokens, and the model learns to predict target tokens conditioned on other ground-truth tokens with a cross-entropy loss training. Existing Transformer-based textto-image generation works $[6,9,36]$ can be extended to support image-to-text generation by exchanging the order of text and image tokens in their input sequences. While they have shown some initial promise, these approaches still exhibit two major challenges for bi-directional generations: information loss caused by the feature discretization process, and error accumulation caused by the cross-entropy loss training. Specifically, first, Transformer-based approaches enable image generation by clustering dense image features into discrete indices as the labels of image tokens $[6,9,36]$, However, this discretization process is harmful to image-to-text generation due to information loss. Second, the cross-entropy loss training in a "teacher-forcing" manner creates a mismatch between training and testing, as the model is only exposed to the training data distribution instead of its own prediction. This "exposure bias" results in error accumulation at test time [37]. Due to these challenges, the typical Transformer-based approach, X-LXMERT [6], generates captions weakly correlated with their source images, and even its text-to-image generation results on automatic metric are worse than its comparing GAN-based approach [53].

We address these challenges with two major designs, i.e., twolevel granularity feature representations and sequence-level training. First, we introduce two-level granularity feature representations, in which we use dense features to reduce information loss for image-to-text generation, and discrete features to enable text-toimage generation. Second, we propose a training strategy that optimizes our model based on its sequence-level prediction instead of token-level predictions to bridge the gap between training and testing. Based on this strategy, we particularly introduce a CLIP-based image-level loss for text-to-image generation, which improves the consistency between generated images and the source text by leveraging a large-scale pre-trained multimodal model CLIP [35]. Moreover, because CLIP learns from a vast amount of image-text pairs on the internet and releases task-specific crowd-sourced labeling, we naturally propose a CLIP-based metric for text-to-image evaluation.

We share most of the Transformer networks for image and text generation tasks and train them iteratively as depicted in Figure 1
(2). This paradigm facilitates image-and-text shared embedding learning, which improves the performance with a half of model size when compared with two separate Transformer models. Compared with a previous Transformer-based approach, X-LXMERT, our approach significantly improves CLIPScore from 72.9 to 77.2 for text-to-image generation, and improves CIDEr-D score from $100.9 \%$ to $122.6 \%$ for image-to-text generation on the MS-COCO dataset. In summary, our contributions are three-fold:

- We present a unified image-and-text generative framework based on a Transformer model with two proposals: (1) twolevel granularity feature representation to avoid information loss ; (2) sequence-level training to mitigate the gap between training and testing.

- We leverage the powerful pre-trained model CLIP to improve text-to-image generation consistency, and to facilitate its evaluation without extra crowd-sourced labeling.

- We conduct automatic and human evaluations that demonstrates our approach significantly improves the quality of both tasks over prior approaches on the MS-COCO dataset.

\section{RELATED WORK}

\subsection{Image-to-Text Generation}

The task of image captioning has attracted increasing attention[2, $7,12,16,26,27,31,40,48-50,52]$. The dominant image captioning models adopt encoder-decoder architectures, which embed images with a CNN encoder, and generate captions with an RNN $[2,40]$ or Transformer decoder [7, 45]. By connecting the encoder and decoder with attention mechanisms, the decoder can selectively integrate relevant information for word generation. Transformer has shown up prominently thanks to its capability in parallel training and modeling global dependency relying entirely on an attention mechanism [45]. Transformer-based large-scale vision-and-language pretraining works over numerous image-text pairs have shown great advances for image captioning [27, 52].

\subsection{Text-to-Image Generation}

Synthesizing images from text descriptions continues to be challenging. Deep generative models based on pixelCNN [44], approximate Langevin sampling [32], variational autoencoders [24], and Generative Adversarial Nets (GANs) [11] have been proposed. Dominant works adopt the GAN framework, which usually consists of a generator and a discriminator to solve a min-max optimization problem, and has shown effectiveness in image generations [38, 47, 51, 53]. Typical works generate images with multiple stages, that they firstly sample low-resolution images, then they gradually upsample and improve images in later stages [38, 47, 51, 53]. Recent large-scale Transformer-based pre-trained works impressed us with their high fidelity images $[9,36]$. DALL-E and CogView greatly advance the quality of text-to-image generation by training transformers with 12-billion and 4-billion parameters on 250 million and 30 million image-text pairs respectively.

\subsection{Bi-directional Image-and-Text Generation}

Image-to-text and text-to-image generation are bi-directional tasks. Huang et al. [17] propose to jointly train an image-to-text generator 
(a) Image and Text Representations

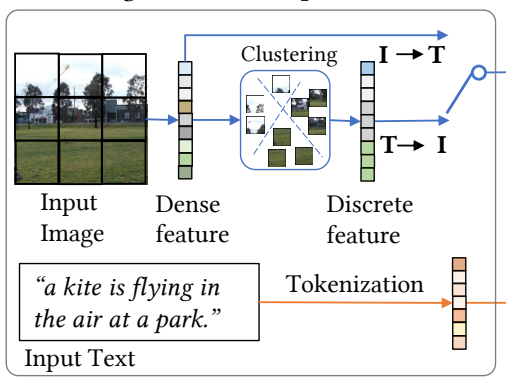

(b) Unified Model (c.1) Token-level training

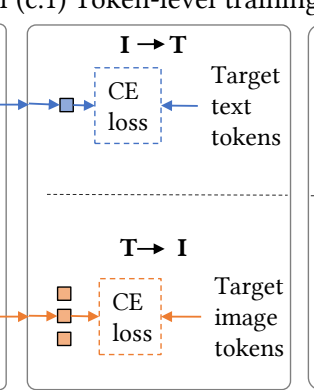

(c.2) Sequence-level training

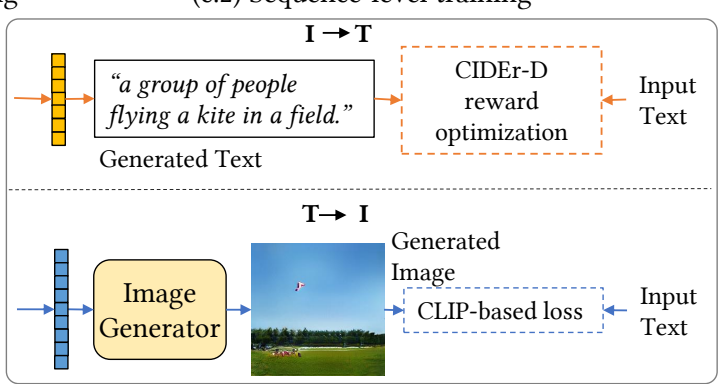

Figure 2: Overview of our framework. We formulate image and text generation tasks as sequence generation tasks. (a) Imageand-text pairs are represented as unified token sequences. Specifically, images are represented as two-level granularity features where we use dense features to reduce information loss for $I->T$ and discrete features to enable image tokenization for $T->I$. (b) The unified Transformer learns multimodal interactions to generate sequences. (c) In addition to the standard token-level training, we introduce sequence-level training strategy to mitigate the mismatch between training and testing. " $I$ " and " $T$ " denote "image" and "text".

based on an LSTM network and a text-to-image generator based on a GAN network to boost both generators by enforcing cycleconsistency in turbo learning. MirrorGAN [34] focus on the text-toimage generation task and uses an off-the-shelf captioning model to regularize the redescription of the generated images to be semantic consistent with the original text. X-LXMERT [6] is a pre-trained model based on Transformer for text-to-image generation, visual question answering, and visual reasoning. Different from these works that use separate bi-directional models, in this paper, we use the same model for image and text generation tasks.

\section{MODEL}

In this section, we introduce our unified multimodal framework and the design of two-level granularity image representation. Figure 2 $(a, b)$ gives an overview of the model.

\subsection{Unified Multimodal Framework}

Our model mainly consists of a unified multimodal Transformer [45], which has a multi-layer architecture and each layer mainly consists of multi-head self-attention and position-wise fully connected feedforward networks. We adopt LXMERT [43] as our Transformerbased architecture following X-LXMERT [6] for a direct comparison, since our proposal is mainly based on feature representation and training mechanism but not on the specific model. LXMERT is a cross-modality Transformer consisting of an object-relation encoder, a language encoder and a cross-modality encoder. We omit an exhaustive background description of the model architecture and refer readers to $[6,43,45]$ for additional details.

To enable both image-to-text and text-to-image generation tasks, we formulate both tasks as sequence generation tasks. Specifically, we firstly pre-process image-and-text pairs into sequences of image tokens and text tokens. Then the Transformer accepts sequences with masked tokens as input, and maps the input to a contextualized representation via attention networks. Lastly, an image or text linear transformation classifier projects the contextualized representation to predicted target tokens. In our model, we share the parameters of most Transformer modules, except for the last classifiers since image and text vocabularies have different sizes and semantics. For text-to-image generation, we use an additional GAN-based image generator to convert the size of $8 \times 8$ image token predictions to a $256 \times 256$ resolution visible image.

Text representation is a combination of the position embedding and the word embedding, where the position refers to the index of the word within the caption, and the word embedding is initialized from pre-trained models like BERT [8] or LXMERT [43].

\subsection{Two-level Granularity Image Representations}

Image representation is a combination of image features and position features. For image features, we split an image into a sequence of uniform grid-level patches, which are effective to learn visual representations for vision-and-language tasks [6, 18, 19, 21]. We propose to use two-level granularity image features. (1) Finegrained dense features: We extract the grid features with a Faster $\mathrm{R}-\mathrm{CNN}$ [39] object detector pre-trained on the Visual Genome dataset [25]. The dense grid features are used as visual inputs for image-to-text generation to reduce the loss of image information. (2) Coarse-grained discrete features: We use discrete clustering features of the dense features to construct the ground-truth labels of visual tokens following X-LXMERT [6]. The discretization process is critical in Transformer-based image generation methods since it is hard to construct a large visual vocabulary for the diverse image pixels or image features, while discretization helps reduce the feature noise and vocabulary size [6,36]. Specifically, we firstly create a visual vocabulary using K-mean clustering, approximate the target visual features via a nearest neighbor search, and then obtain the cluster indices and discrete grid features for each image. Naturally, the position feature of each grid is a 2-D embedding of the grid-level bounding box positions.

\section{TRAINING}

We formulate the bi-directional image and text generation tasks as sequence generation tasks and iteratively train the Transformer. 
Sequence models are usually trained using the cross-entropy loss in a "Teacher-Forcing" manner, where ground truth tokens are given in each step of training. Since the model is only exposed to the training data distribution instead of its own predictions, a mismatch between training and testing named "exposure bias" is created and results in error accumulation at test time [37]. To tackle this problem, we design a two-stage training strategy.

- Stage 1. Token-level Training. This stage aims to generate fluent captions and realistic images by "teacher-forcing" training on word level or grid level in each step for imageto-text or text-to-image generation respectively.

- Stage 2. Sequence-level Training. This stage further optimizes the model with generated text or image sequence to bridge the gap between training and testing.

Figure 2 (c) gives an overview of the training process.

\subsection{Image-to-Text Generation}

4.1.1 Stage 1. Word-level Training. In the first stage, we aim to train an image caption generator in a "Teacher-Forcing" manner similar to training a uni-directional autoregressive transformer. The model is trained to maximize the likelihood of the next ground-truth word given the previous ground-truth words and visual context using back-propagation. We denote parameters of Transformer model with $\theta$ and minimize the following cross-entropy loss:

$$
L_{\text {Tok }}^{T}(\theta)=-\sum_{t=1}^{L} \log p_{\theta}\left(\mathbf{y}_{l} \mid \mathbf{y}_{1: l-1}, \mathbf{X}\right),
$$

where $\mathbf{X}=\mathbf{x}_{1: M}$ and $\mathrm{Y}=\mathbf{y}_{1: L}$ are the sequences of ground truth image and text tokens respectively. $M$ and $L$ are the sequence length for image and text sequences respectively.

4.1.2 Stage 2. Sentence-level Training. To alleviate the exposure bias, we adopt the reinforcement learning algorithm of Self-Critical Sequence Training (SCST) [40] to directly optimize non-differentiable sentence-level metrics (e.g., CIDEr-D [46]). We minimize the negative expected score function $r$ of CIDEr-D metric:

$$
L_{S e q}^{T}(\theta)=-\mathbf{E}_{\hat{\mathbf{y}}_{1: L} \sim p_{\theta}}\left[r\left(\hat{\mathbf{y}}_{1: L}\right)\right],
$$

where $\hat{\mathbf{Y}}=\hat{\mathbf{y}}_{1: L}$ denotes the sampled text tokens.

\subsection{Text-to-Image Generation}

4.2.1 Stage 1. Grid-level Training. The training target is to maximize the likelihood of the target visual tokens given the other ground-truth visual tokens and text context, which is similar to the training target of the first stage of image-to-text generation. However, text-to-image direction aims to predict multiple tokens instead of one token at each step, by minimizing the cross-entropy loss overall $M^{\prime}$ masked tokens:

$$
L_{T o k}^{I}(\theta)=-\sum_{m=1}^{M^{\prime}} \log p_{\theta}\left(\mathbf{x}_{m} \mid \mathbf{x}_{\backslash M^{\prime}}, \mathbf{Y}\right),
$$

where $\mathbf{x}_{\backslash M^{\prime}}$ denotes the sequence of tokens excluding the masked tokens. We follow X-LXMERT to use this training strategy to enables a non-autoregressive sampling strategy, i.e., mask-predict- $\mathrm{k}$ strategy [10]. In this way, only a few sampling steps (e.g., $k=4$ ) are needed to generate all visual tokens of an image, which enjoys a faster inference speed for broader industrial applications.

4.2.2 Stage 2. Image-level Training. Although the cross-entropy loss in grid-level training has shown initial promise, there remain two major issues. First, the loss imposes restrictive supervision on each generated image to regard one reference image as a "gold label". This violates the one-to-many property of text-to-image mapping, where a caption can correspond to many feasible images. Second, the loss is based on image grid indices or features, which ignores the relations across grids.

To tackle these issues, we propose to directly optimize the model towards generating an image that is more semantic consistent with the source caption instead of one reference image. To achieve this, we leverage a large-scale pre-trained multimodal model CLIP [35] to score the image-text consistency. This is desirable since CLIP is a general model pre-trained on $400 \mathrm{M}$ image-text pairs from the web and exhibits unparalleled zero-shot transferable ability in a great variety of classification benchmarks. DALLE has used CLIP to re-rank its generated images with CLIP-based scores as an offline post-process [36], while we are the first to leverage a CLIP-based loss to directly regularize the training for text-to-image generation.

Specifically, we extract image and text embedding from the CLIP and calculate their cosine similarity to obtain CLIP-based loss:

$$
L_{\text {clip }}^{I}(\theta)=-\max (\cos (\mathcal{I}(\hat{\mathrm{X}}), \mathcal{T}(\mathrm{Y})), 0),
$$

where $\mathcal{I}(\cdot)$ and $\mathcal{T}(\cdot)$ are the image and text embedding extraction networks of CLIP. Note that the image embedding is also a Gumbel-Softmax approximation [20] to support model optimization with back-propagation. Learning from CLIP has several potential strengths. It releases the crowd-sourced labeling, and naturally conforms to the one-to-many property of text-to-image mapping thanks to learning from the vast amount of image-text pairs on the internet. Moreover, it connects text with the whole images instead of image grids to consider the relations across grids, thus it encourages higher semantic consistency between images and text.

We also have experimented with a grid feature similarity loss, a pixel-wise loss and a perceptual loss [22], but we do not observe much improvement in our preliminary experiments. Due to the high variance property of sampling results of non-autoregressive sampling strategy, we conduct this second stage training accompanying with the first stage training iteratively, since the first stage of "teacher-forcing" training can encourage the sampling coherency.

\section{EXPERIMENTS}

\subsection{Experimental Setup}

MS-COCO Dataset [29]. We evaluate our proposed method on the popular MS-COCO dataset. It is collected using Amazon Mechanical Turk (AMT) with five annotated captions for each image. MSCOCO dataset's official splits include 82,783/40,504/40,775 images for training/validation/testing set respectively. We train our model on the train split and evaluate our model on the validation split by randomly sampling 30,000 images following most text-to-image generation works $[6,53]$. For image-to-text generation (image captioning), we follow most captioning works to evaluate our model on the Karpathy test split, which is a subset of the validation set consisting of 5,000 images. Our results on image captioning are not 
Table 1: Ablations on image-to-text (I2T) and text-to-image (T2I) tasks on MSCOCO test set. (1) Our unified single Transformer model exhibits comparable performance to task-specific Transformers with half of model parameters. (2) Our two-level granularity image representation inherits the advantages from both discrete features for T2I, and dense features for I2T. (3) Our proposed sequence-level training significantly improves both tasks. (4) Our proposed CLIP-based loss improves T2I by improving the consistency between generated images and the source text.

\begin{tabular}{|c|c|c|c|c|c|c|c|c|c|c|}
\hline \multirow{3}{*}{ Model } & \multirow{3}{*}{ Parameters $\downarrow$} & \multicolumn{5}{|c|}{ Image-to-Text Generation } & \multicolumn{4}{|c|}{ Text-to-Image Generation } \\
\hline & & $\mathrm{B} \cap 4 \uparrow$ & $M \uparrow$ & $\mathrm{B} \uparrow$ & $C \uparrow$ & $\mathrm{S} \uparrow$ & CIJPScrere & FID! & R-precision & hard/easy) \\
\hline & & $D @ 4$ & $\mathrm{N1}$ & $\mathrm{K}_{\mid}$ & 4 & 31 & CLIPscore & FID $\downarrow$ & ViLBERT $\uparrow$ & $\mathrm{CLIP} \uparrow$ \\
\hline Ours & $228 \mathrm{M}$ & 37.3 & 28.2 & 57.9 & 122.6 & 21.9 & 77.2 & 29.9 & $37.7 / 59.2$ & $40.7 / 69.9$ \\
\hline w/o unified architecture & $456 \mathrm{M}$ & 37.4 & 28.2 & 58.0 & 122.3 & 22.0 & 76.5 & 30.2 & $37.0 / 59.6$ & $40.3 / 68.9$ \\
\hline \multicolumn{11}{|l|}{ w/o two-level features } \\
\hline Dense feature & $228 \mathrm{M}$ & 37.2 & 28.2 & 57.9 & 122.2 & 22.0 & 75.7 & 34.9 & $32.9 / 51.2$ & $38.6 / 61.9$ \\
\hline Discrete feature & $228 \mathrm{M}$ & 34.7 & 27.0 & 56.0 & 114.3 & 20.7 & 76.9 & 30.2 & $37.0 / 59.1$ & $40.7 / 69.3$ \\
\hline w/o sequence-level training & $228 \mathrm{M}$ & 32.2 & 26.9 & 54.8 & 107.9 & 20.2 & 73.4 & 33.5 & $33.3 / 51.1$ & $35.5 / 63.0$ \\
\hline w/o CLIP-based loss & $228 \mathrm{M}$ & 37.6 & 28.3 & 58.1 & 122.5 & 22.0 & 72.5 & 40.1 & $30.7 / 47.6$ & $34.2 / 59.0$ \\
\hline
\end{tabular}

directly comparable to other image captioning models since they are trained with a larger split (113,287 vs. 82,783) and are expected to score higher. Moreover, we use grid-based $8 \times 8$ features for a fair comparison with X-LXMERT, while this feature is weaker than the 100 region-based feature used by standard image captioning works. Implementation Details. Our code is available online ${ }^{1}$. For details on model architecture, we initialize our model from the pre-trained X-LXMERT model [6] for a direct comparison, which adopts the architecture of LXMERT [43] and is pre-trained with MS-COCO Captions [29], Visual Genome [25] and VQA [3] datasets. We adopt an image generator consisting of convolutional layers and trained with Generative Adversarial Networks (GAN) [11] method following X-LXMERT. Also, we directly use the pre-trained image generator provided by X-LXMERT for a fair comparison ${ }^{2}$. We limit the length of a text caption to $L=17$ tokens, and the grid size of each image is $M=8 \times 8$. We use a vocabulary of 30,522 tokens for text words, and a vocabulary of 10,000 tokens for image grids.

For details on training, we use the AdamW [23] optimizer with beta coefficients of 0.9 and 0.999 , and a weight decay of $1 \mathrm{e}-2$ following X-LXMERT. Both image-to-text or text-to-image generation tasks take 100,000 iterations in the first or second stage training. For the first stage training, we linearly warm up the learning rate from 0 to $5 \mathrm{e}-5$ over the first $5 \%$ iterations, and cosine decay it in the rest of training steps following X-LXMERT. Since the second stage of training is initialized from the first stage, we use a fixed smaller learning rate of $1 \mathrm{e}-6$. We train the first stage with a batch size of 256, and the second stage of 160 empirically. We use a label smoothing of 0.2 [42], and a gradient clipping threshold of 1.0. We adopt mixed-precision training to reduce memory cost and speed up the training procedure.

\subsection{Evaluation Criteria}

Image-to-Text Generation. We report five commonly-used automatic evaluation metrics for the image captioning task: BLEU@N [33] $(\mathrm{N}=1,4)$, ROUGE-L [28], MEREOR [4], CIDEr-D [46] and SPICE [1], which are denoted by $\mathrm{B} @ \mathrm{~N}, \mathrm{M}, \mathrm{R}, \mathrm{C}$, and $\mathrm{S}$ for abbreviation.

\footnotetext{
${ }^{1}$ https://github.com/researchmm/generate-it

${ }^{2}$ https://github.com/allenai/x-lxmert
}

Text-to-Image Generation. We evaluate text-to-image generation from three aspects: (1) Fréchet Inception Distance (FID) [14] compares the distribution of generated images with the distribution of real images. A lower FID implies a closer distance between generated image distribution and real-world image distribution. (2) R-precision [47] ranks retrieval results to evaluate whether the generated image is well conditioned on the given text. We follow X-LXMERT to use two variants of R-precision to fully evaluate our model. The easy variant randomly samples negatives among the test caption set. The hard variant swaps a word in a caption with another word within the same category. To compute the image and text similarity for ranking, X-LXMERT uses ViLBERT-MT [30], an off-the-shelf multimodal network based on object-level and word-level representations. We propose to use CLIP-based representations, as a complementary R-precision evaluation metric from global image and text levels. (3) CLIPScore. Since the R-precision cannot directly reflect the individual image-and-text consistency, we propose to use a CLIP-based score, which calculates the cosine similarity between the image and text representations from CLIP [35], as a complementary metric for text-to-image evaluation. CLIP is a powerful multimodal pre-trained model to evaluate the image-text consistency, where a CLIP-based metric (i.e., CLIPScore) has been proposed by Jack et al. to evaluate image captioning models without reference captions [13]. Instead, we extend CLIPScore to evaluate image generation models.

We do not use Inception score (IS) [41], since it overfits within the context of text-to-image generation and can be manipulated to achieve much higher scores using simple tricks $[5,15]$.

\subsection{Ablation Studies}

We conduct ablation studies to evaluate our designs. Except for the ablated component, we use the same model, dataset and other implementation details. Results are shown in Table 1.

What is the benefit of unified architecture? We evaluate the impact of training a bi-directional unified Transformer by comparing our model to two separate Transformer models for image-to-text and text-to-image generations. For text-to-image generation task, our unified model outperforms the separate model on most metrics. 
Table 2: Comparisons with existing approaches on MS-COCO test set. We generate images (or captions) for DM-GAN [53], $X$-LXMERT [6] with their released codes and models, and evaluate the images (or captions) in the same way as ours and $X$ LXMERT-FT for more direct comparison. For BUTD [2] and Turbo-RL [17], we report the numbers recorded in their published papers. "-" indicates the detail is not reported. "N/A" is the abbreviation of "not applicable". "I", "T", "Arch" and "TF" denote "image", "text", "architecture" and "Transformer" respectively.

\begin{tabular}{|c|c|c|c|c|c|c|c|c|c|c|c|c|}
\hline \multirow{3}{*}{ Direction } & \multirow{3}{*}{ Model } & \multicolumn{6}{|c|}{ Image-to-Text Generation } & \multicolumn{5}{|c|}{ Text-to-Image Generation } \\
\hline & & \multirow{2}{*}{ Arch } & \multirow{2}{*}{$\mathrm{B} @ 4 \uparrow$} & \multirow{2}{*}{$\mathrm{M} \uparrow$} & \multirow{2}{*}{$\mathrm{R} \uparrow$} & \multirow{2}{*}{$\mathrm{C} \uparrow$} & \multirow{2}{*}{$\mathrm{S} \uparrow$} & \multirow{2}{*}{ Arch } & \multirow{2}{*}{ CLIPScore $\uparrow$} & \multirow{2}{*}{ FID $\downarrow$} & \multicolumn{2}{|c|}{ R-precision (hard/easy) } \\
\hline & & & & & & & & & & & ViLBERT $\uparrow$ & $\mathrm{CLIP} \uparrow$ \\
\hline $\mathrm{T}->\mathrm{I}$ & DM-GAN & \multicolumn{6}{|c|}{$\mathrm{N} / \mathrm{A}$} & $\mathrm{CNN}$ & 72.3 & 35.9 & $32.7 / 58.4$ & $34.4 / 69.1$ \\
\hline $\mathrm{I}->\mathrm{T}$ & $\mathrm{BUTD}^{\dagger}$ & RNN & 36.3 & 27.7 & 56.9 & 120.1 & 21.4 & \multicolumn{5}{|c|}{$\mathrm{N} / \mathrm{A}$} \\
\hline \multirow{4}{*}{$\mathrm{I}<->\mathrm{T}$} & Turbo-RL $^{\dagger}$ & RNN & 31.6 & 21.9 & 49.8 & 74.8 & 17.5 & $\mathrm{CNN}$ & - & - & - & - \\
\hline & X-LXMERT & $\mathrm{TF}$ & 15.2 & 22.7 & 42.2 & 41.0 & 16.6 & $\mathrm{TF}$ & 72.9 & 37.0 & $33.1 / 49.8$ & $36.3 / 60.1$ \\
\hline & X-LXMERT-FT & $\mathrm{TF}$ & 30.2 & 25.8 & 53.3 & 100.9 & 19.1 & $\mathrm{TF}$ & 73.3 & 33.9 & $33.1 / 50.7$ & $35.1 / 62.3$ \\
\hline & Ours & $\mathrm{TF}$ & 37.3 & 28.2 & 57.9 & 122.6 & 21.9 & $\mathrm{TF}$ & 77.2 & 29.9 & $37.7 / 59.2$ & $40.7 / 69.9$ \\
\hline
\end{tabular}

Note that the models denoted with ${ }^{\dagger}$ are trained on 113,287 images, and are expected to score higher on image-to-text generation than other models trained on 82,783 images.

They exhibit comparable performance on image-to-text generation task. Moreover, the parameter size of our unified model is a half of two separate models' parameter size. We expect smaller Transformer could further decrease the model size. Thus our model significantly benefits industrial application towards optimizing storage utilization.

Do two-level granularity image features help? As we have introduced in Section 3.2, the common practice for image-to-text or text-to-image generation is using images' original dense feature or their discrete form respectively. To conduct bi-directional generations in a unified model, we separately train three models with inputs of dense feature, discrete feature and our two-level granularity feature. Note that when taking dense feature as input, the model still learns to predict discrete image tokens instead of dense features for text-to-image generation, otherwise the model would fail to generate normal images validated by X-LXMERT [6]. However, this creates a mismatch between training and testing. Results show that dense feature performs much worse than our two-level feature in text-to-image generation, despite its good performance in image-to-text generation. Contrarily, using only the discrete feature performs much better than dense feature in text-toimage generation, however, not in image-to-text generation. Our design of two-level granularity feature inherits the advantages of dense features on image-to-text generation, and discrete features on text-to-image generation, and thus performs well on both tasks. What is the impact of the sequence-level training? We show that removing the sequence-level training and leaving a single stage of token-level training hurts performance significantly on both tasks. For text-to-image generation, the CIDEr-D score drops from $122.6 \%$ to $107.9 \%$; for image-to-text generation, the CLIPScore drops from 77.2 to 73.4 . The results illustrate the effectiveness of sequence-level training to mitigate the "exposure bias" mismatch between training and testing.

Do we need the CLIP-based loss? To verify the effect of our proposed CLIP-based loss for text-to-image generation in the imagelevel training, we replace the CLIP-based loss with a mean squared error (squared L2 norm) loss based on grid feature similarity. From the results, we see that it hurts performance significantly on all textto-image evaluation metrics. The results highlight the effectiveness of CLIP-based loss to improve the semantic consistency between generated images and source text. The performance of image-totext generation is not influenced, demonstrating the robustness of our model.

\subsection{Quantitative Results}

We compare our approach with typical published uni-directional approaches with task-specific designs, and bi-directional approaches with or without task-specific designs as followed. We choose models that are mainly trained with MS-COCO dataset as ours.

- DM-GAN [53] is a typical text-to-image generation model with a GAN-based framework. It progressively generates images from low-resolution to high-resolution synthesis, and adaptively refines images with a memory module.

- BUTD [2] is a image-to-text generation model with an encoderdecoder architecture based on CNN-LSTM networks. It uses a combined bottom-up and top-down attention mechanism to enable attention on objects.

- Turbo-RL [17] is a bi-directional image and text generation model. It is jointly trained with an LSTM network and a GAN network to boost both generators by enforcing cycle consistency in turbo learning. Since the authors did not report text-to-image generation results relevant to our metric, and we cannot find released codes, their results are not reported.

- X-LXMERT [6] is a vision-and-language pre-trained model for text-to-image generation, visual question answering, and visual reasoning tasks. X-LXMERT remains image captioning capability to sample sentences by masking and predicting word tokens. As the authors did not report their image-totext results, we generate captions with a prefix word "A" as they suggested by their released code and model.

- X-LXMERT-FT is a model separately fine-tuned on imageto-text or text-to-image generation task from the released pre-trained X-LXMERT model with standard cross-entropy loss training. This provides a direct comparison with our model on feature representation and training strategy. 


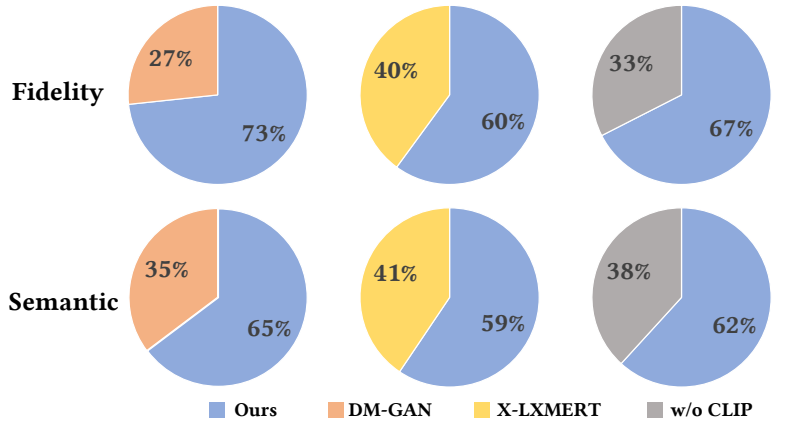

Figure 3: Human evaluation on text-to-image generation between our model, DM-GAN [53], X-LXMERT [6] and our ablated model trained without CLIP-based loss (denoted by "w/o CLIP"). Our model clearly outperforms others in both aspects of fidelity and semantic of images.

Table 2 provides comprehensive results. Across two tasks, our model achieves significant performance gains over comparing methods in all metrics. For task-specific uni-directional models, our model outperforms the typical image-to-text model BUTD by $2.5 \%$ CIDEr-D score, despite being trained with much fewer images. Our model also outperforms typical text-to-image model DM-GAN by 4.9 and 6.0 on CLIPScore and FID respectively. The task-specific bi-directional model Turbo-RL achieves 74.8\% CIDEr-D score on MSCOCO Karpathy test set, which is far from satisfactory. The inferior results achieved by task-specific works may indicate the limitation of RNN caption decoder or $\mathrm{CNN}$ image generator.

For Transformer-based approaches, the pre-trained X-LXMERT model achieves $41.0 \%$ in CIDEr-D score and 15.2\% in BLEU@4, indicating the original model is not able to generate very accurate and fluent captions for MS-COCO dataset. This large gap may be due to the mismatch between X-LXMERT's pre-training dataset (MS-COCO [29], VG [25] and VQA [3]) and downstream dataset (MS-COCO), so we fine-tune X-LXMERT with the standard crossentropy loss training denoted by X-LXMERT-FT for fair comparisons. X-LXMERT-FT do obtain large gains from $41.0 \%$ to $100.9 \%$ in CIDEr-D metric for image-to-text generation task, and from 37.0 to 33.9 in FID score for text-to-image generation task. However, there is still a gap between X-LXMERT-FT and the task-specific models, and there is little gain over semantic consistency-aware metrics CLIPScore and R-precision. Our model mitigates this gap by significantly improving CIDEr-D score from $100.9 \%$ to $122.6 \%$. Moreover, our model surpasses X-LXMERT-FT by 3.9 points in CLIPScore, 4.6/8.5 points in VilBERT-based R-precision, and 5.6/7.6 points in CLIP-based R-precision. The results confirm the superiority of our model, and validates that a unified Transformer could result in similar breakthroughs in both image-to-text and text-toimage generations.

\subsection{Human Evaluation}

To better evaluate the quality of generated images, we conduct a human evaluation to compare our method with existing works and visualize results in Figure 3. Specifically, we choose DMGAN [53] and X-LXMERT [6] for comparisons, which are the best-performing
GAN-based and Transformer-based published works with released models, respectively. We also compare with our ablated model without CLIP-based loss (denoted by “w/o CLIP”). We randomly sample 300 captions from MSCOCO test set and generate images from each caption by different models. During the evaluation, we provide a caption and an image pair generated by our model and other models in random order. We invite ten volunteers who are proficient in English with over ten years of learning experiences and ask to select the one that (1) shows higher fidelity and (2) better matches the source caption semantically. As we can see from Figure 3, our model significantly surpasses DM-GAN, X-LXMERT and "w/o CLIP" by about $37 \%, 19 \%$ and $29 \%$ on an average of fidelity and semantic scores. The results validate the superiority of our model.

\subsection{Qualitative Examples}

Visual inspection of generated images and captions in Figure 4 convincingly shows the large quality improvement. For text generation, our model performs comparable with BUTD, which is unidirectional approach. Compared with X-LXMERT, our model generates more accurate captions with better rationality. Some made-up objects like "zebra" and "red shirt" do not appear in our generated captions. For image generation, our model generates images with more realistic objects and higher consistency with texts. Our model outperforms DM-GAN by a large margin on fidelity. Compared with X-LXMERT and w/o CLIP, our model learns better alignment with texts from CLIP-based loss (notice people on the beach, ship in the water, kite in the air.). Compared with the discrete-feature-based model, our model generates more realistic and smooth images.

\section{CONCLUSION}

In this work, we propose a unified multimodal Transformer for bi-directional image-and-text generation tasks. Our proposal alleviates the expensive design efforts of task-specific models, and optimizes storage utilization compared to the design of two separate models for bi-directional tasks. To tackle the challenges of Transformer-based image-and-text generative models, we design a two-level granularity feature representation and a sequence-level training strategy. The two-level granularity feature representation addresses the information loss issue caused by feature discretization process. The sequence-level training strategy address the error accumulation in test time caused by the cross-entropy training. Sufficient qualitative and quantitative experiments have shown the effectiveness of our approach. With these benefits, our model could facilitate industrial applications for multimodal interactions with desirable performance.

\section{ACKNOWLEDGEMENT}

This work was supported by the NSFC (U1811461), the Guangdong Natural Science Foundation (2018B030312002), and the Program for Guangdong Introducing Innovative and Entrepreneurial Teams under Grant NO.2016ZT06D211.

We would like to acknowledge Yiheng Xu and Zhicheng Huang for the helpful discussions. 


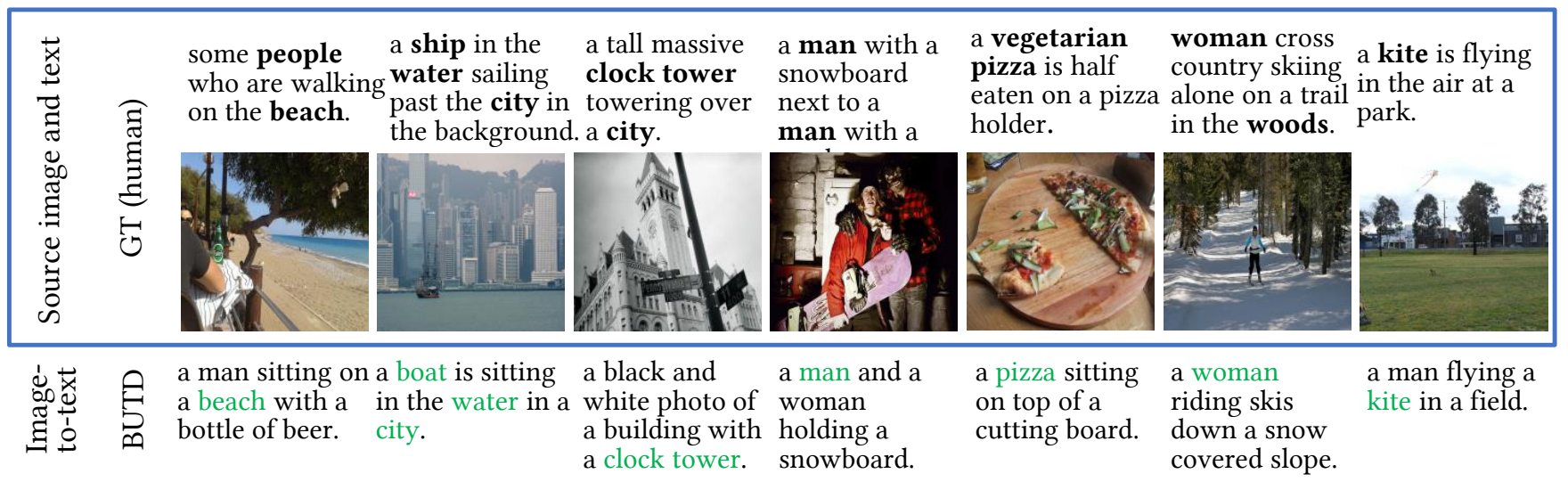

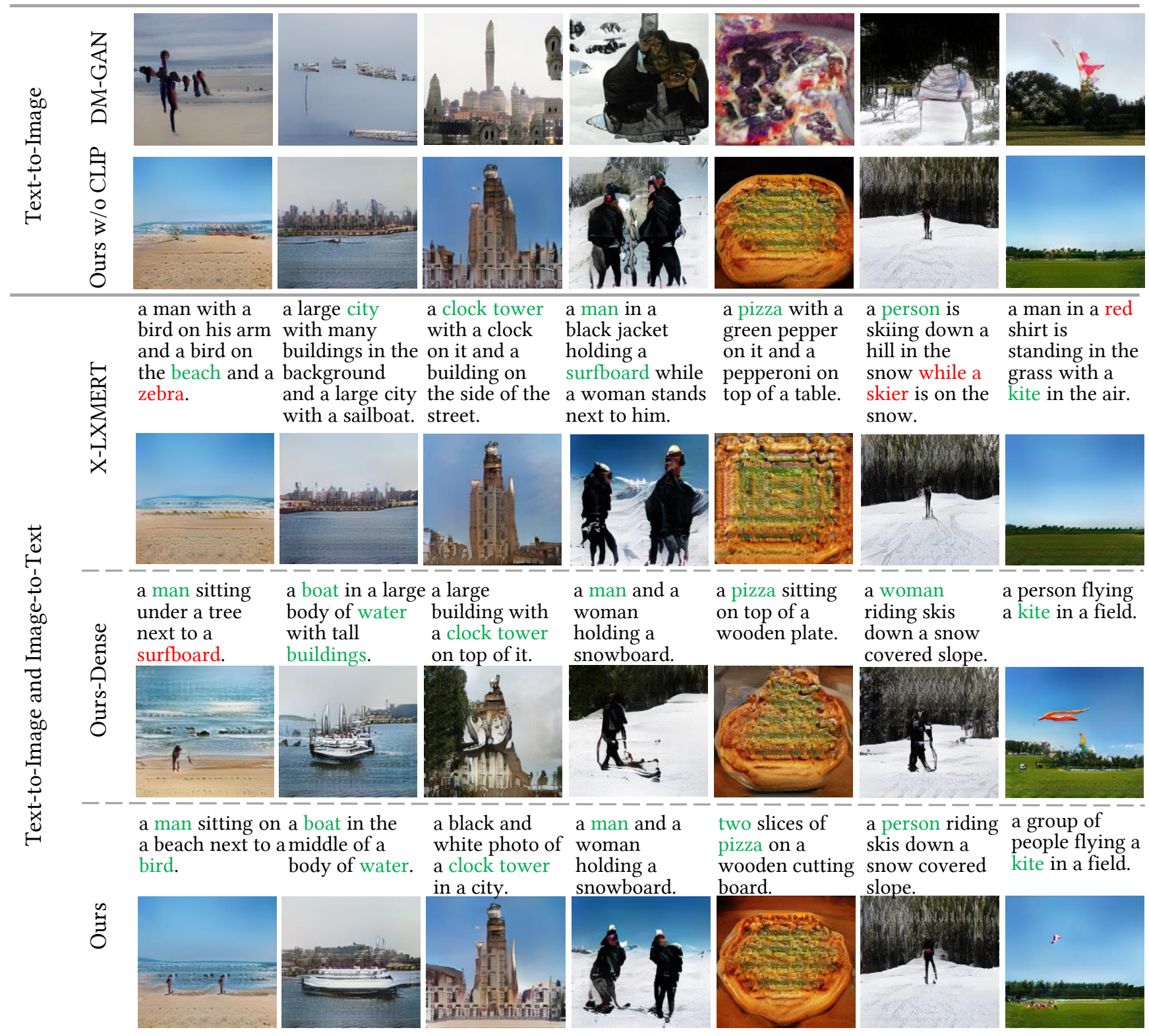

Figure 4: Examples of text-to-image and image-to-text generation results generated by different models from human written captions or real images (human ground-truths in blue box). Right and wrong expressions in captions are highlighted in green and red respectively. Our model generates the most accurate and real images and text when compared with others. 


\section{REFERENCES}

[1] Peter Anderson, Basura Fernando, Mark Johnson, and Stephen Gould. 2016 Spice: Semantic propositional image caption evaluation. In European Conference on Computer Vision. 382-398.

[2] Peter Anderson, Xiaodong He, Chris Buehler, Damien Teney, Mark Johnson, Stephen Gould, and Lei Zhang. 2018. Bottom-up and top-down attention for image captioning and visual question answering. In Proceedings of the IEEE conference on computer vision and pattern recognition. 6077-6086.

[3] Stanislaw Antol, Aishwarya Agrawal, Jiasen Lu, Margaret Mitchell, Dhruv Batra C Lawrence Zitnick, and Devi Parikh. 2015. Vqa: Visual question answering. In Proceedings of the IEEE international conference on computer vision. 2425-2433.

[4] Satanjeev Banerjee and Alon Lavie. 2005. METEOR: An automatic metric for MT evaluation with improved correlation with human judgments. In Proceedings of the acl workshop on intrinsic and extrinsic evaluation measures for machine translation and/or summarization. 65-72.

[5] Shane Barratt and Rishi Sharma. 2018. A note on the inception score. ICML 2018 Workshop on Theoretical Foundations and Applications of Deep Generative Models (2018).

[6] Jaemin Cho, Jiasen Lu, Dustin Schwenk, Hannaneh Hajishirzi, and Aniruddha Kembhavi. 2020. X-LXMERT: Paint, Caption and Answer Questions with MultiModal Transformers. In EMNLP. 8785-8805.

[7] Marcella Cornia, Matteo Stefanini, Lorenzo Baraldi, and Rita Cucchiara. 2020. M ${ }^{2}$ : Meshed-Memory Transformer for Image Captioning. In CVPR.

[8] Jacob Devlin, Ming-Wei Chang, Kenton Lee, and Kristina Toutanova. 2019. BERT: Pre-training of Deep Bidirectional Transformers for Language Understanding. In Proceedings of the 2019 Conference of the North American Chapter of the Association for Computational Linguistics: Human Language Technologies, Volume 1 (Long and Short Papers). 4171-4186.

[9] Ming Ding, Zhuoyi Yang, Wenyi Hong, Wendi Zheng, Chang Zhou, Da Yin, Junyang Lin, Xu Zou, Zhou Shao, Hongxia Yang, and Jie Tang. 2021. CogView: Mastering Text-to-Image Generation via Transformers.

[10] Marjan Ghazvininejad, Omer Levy, Yinhan Liu, and Luke Zettlemoyer. 2019 Mask-Predict: Parallel Decoding of Conditional Masked Language Models. In EMNLP. 6114-6123.

[11] Ian Goodfellow, Jean Pouget-Abadie, Mehdi Mirza, Bing Xu, David Warde-Farley, Sherjil Ozair, Aaron Courville, and Yoshua Bengio. 2014. Generative Adversarial Nets. In Advances in Neural Information Processing Systems, Vol. 27.

[12] Longteng Guo, Jing Liu, Jinhui Tang, Jiangwei Li, Wei Luo, and Hanqing Lu. 2019. Aligning Linguistic Words and Visual Semantic Units for Image Captioning. In Proceedings of the 27th ACM International Conference on Multimedia. 765-773.

[13] Jack Hessel, Ari Holtzman, Maxwell Forbes, Ronan Le Bras, and Yejin Choi. 2021 CLIPScore: A Reference-free Evaluation Metric for Image Captioning. arXiv preprint arXiv:2104.08718 (2021).

[14] Martin Heusel, Hubert Ramsauer, Thomas Unterthiner, Bernhard Nessler, and Sepp Hochreiter. 2017. GANs Trained by a Two Time-Scale Update Rule Converge to a Local Nash Equilibrium. In Advances in Neural Information Processing Systems, Vol. 30

[15] Tobias Hinz, Stefan Heinrich, and Stefan Wermter. 2020. Semantic Object Accuracy for Generative Text-to-Image Synthesis. IEEE Transactions on Pattern Analysis and Machine Intelligence (2020).

[16] Lun Huang, Wenmin Wang, Jie Chen, and Xiao-Yong Wei. 2019. Attention on attention for image captioning. In Proceedings of the IEEE International Conference on Computer Vision. 4634-4643.

[17] Qiuyuan Huang, Pengchuan Zhang, Dapeng Wu, and Lei Zhang. 2018. Turbo learning for CaptionBot and DrawingBot. In Proceedings of the 32nd International Conference on Neural Information Processing Systems. 6456-6466.

[18] Zhicheng Huang, Zhaoyang Zeng, Yupan Huang, Bei Liu, Dongmei Fu, and Jianlong Fu. 2021. Seeing Out of tHe bOx: End-to-End Pre-training for VisionLanguage Representation Learning. In The IEEE Conference on Computer Vision and Pattern Recognition (CVPR).

[19] Zhicheng Huang, Zhaoyang Zeng, Bei Liu, Dongmei Fu, and Jianlong Fu. 2020 Pixel-bert: Aligning image pixels with text by deep multi-modal transformers. arXiv preprint arXiv:2004.00849 (2020)

[20] Eric Jang, Shixiang Gu, and Ben Poole. 2016. Categorical reparameterization with gumbel-softmax. arXiv preprint arXiv:1611.01144 (2016).

[21] Huaizu Jiang, Ishan Misra, Marcus Rohrbach, Erik Learned-Miller, and Xinlei Chen. 2020. In defense of grid features for visual question answering. In Proceedings of the IEEE/CVF Conference on Computer Vision and Pattern Recognition. 10267-10276.

[22] Justin Johnson, Alexandre Alahi, and Li Fei-Fei. 2016. Perceptual losses for realtime style transfer and super-resolution. In European conference on computer vision. 694-711.

[23] Diederik P Kingma and Jimmy Ba. 2015. Adam: A method for stochastic optimization. In International Conference on Learning Representations.

[24] Diederik P Kingma and Max Welling. 2014. Auto-Encoding Variational Bayes. In ICLR.

[25] Ranjay Krishna, Yuke Zhu, Oliver Groth, Justin Johnson, Kenji Hata, Joshua Kravitz, Stephanie Chen, Yannis Kalantidis, Li-Jia Li, David A Shamma, et al.
2017. Visual genome: Connecting language and vision using crowdsourced dense image annotations. International fournal of Computer Vision 123, 1 (2017), 32-73.

[26] Guang Li, Linchao Zhu, Ping Liu, and Yi Yang. 2019. Entangled Transformer for Image Captioning. In Proceedings of the IEEE International Conference on Computer Vision. 8928-8937.

[27] Xiujun Li, Xi Yin, Chunyuan Li, Pengchuan Zhang, Xiaowei Hu, Lei Zhang, Lijuan Wang, Houdong Hu, Li Dong, Furu Wei, et al. 2020. Oscar: Object-semantics aligned pre-training for vision-language tasks. In European Conference on Computer Vision. Springer, 121-137.

[28] Chin-Yew Lin. 2004. Rouge: A package for automatic evaluation of summaries. In Text Summarization Branches Out: Proceedings of the Association for Computational Linguistics Workshop. 74-81.

[29] Tsung-Yi Lin, Michael Maire, Serge Belongie, James Hays, Pietro Perona, Deva Ramanan, Piotr Dollár, and C Lawrence Zitnick. 2014. Microsoft coco: Common objects in context. In European conference on computer vision. Springer, 740-755.

[30] Jiasen Lu, Dhruv Batra, Devi Parikh, and Stefan Lee. 2019. Vilbert: Pretraining task-agnostic visiolinguistic representations for vision-and-language tasks. In Advances in Neural Information Processing Systems. 13-23.

[31] Jiasen Lu, Jianwei Yang, Dhruv Batra, and Devi Parikh. 2018. Neural baby talk. In Proceedings of the IEEE conference on computer vision and pattern recognition. 7219-7228.

[32] Anh Nguyen, Jeff Clune, Yoshua Bengio, Alexey Dosovitskiy, and Jason Yosinski. 2017. Plug \& play generative networks: Conditional iterative generation of images in latent space. In Proceedings of the IEEE Conference on Computer Vision and Pattern Recognition. 4467-4477.

[33] Kishore Papineni, Salim Roukos, Todd Ward, and Wei-Jing Zhu. 2002. BLEU: a method for automatic evaluation of machine translation. In Proceedings of the 40th annual meeting on association for computational linguistics. 311-318.

[34] Tingting Qiao, Jing Zhang, Duanqing Xu, and Dacheng Tao. 2019. Mirrorgan: Learning text-to-image generation by redescription. In Proceedings of the IEEE/CVF Conference on Computer Vision and Pattern Recognition. 1505-1514.

[35] Alec Radford, Jong Wook Kim, Chris Hallacy, Aditya Ramesh, Gabriel Goh, Sandhini Agarwal, Girish Sastry, Amanda Askell, Pamela Mishkin, Jack Clark, et al. 2021. Learning transferable visual models from natural language supervision. arXiv preprint arXiv:2103.00020 (2021).

[36] Aditya Ramesh, Mikhail Pavlov, Gabriel Goh, Scott Gray, Chelsea Voss, Alec Radford, Mark Chen, and Ilya Sutskever. 2021. Zero-shot text-to-image generation. arXiv preprint arXiv:2102.12092 (2021).

[37] Marc'Aurelio Ranzato, Sumit Chopra, Michael Auli, and Wojciech Zaremba. 2016. Sequence Level Training with Recurrent Neural Networks. In ICLR.

[38] Scott Reed, Zeynep Akata, Xinchen Yan, Lajanugen Logeswaran, Bernt Schiele, and Honglak Lee. 2016. Generative adversarial text to image synthesis. In International Conference on Machine Learning. PMLR, 1060-1069.

[39] Shaoqing Ren, Kaiming He, Ross Girshick, and Jian Sun. 2015. Faster r-cnn: Towards real-time object detection with region proposal networks. In Advances in neural information processing systems. 91-99.

[40] Steven J Rennie, Etienne Marcheret, Youssef Mroueh, Jerret Ross, and Vaibhava Goel. 2017. Self-critical sequence training for image captioning. In Proceedings of the IEEE Conference on Computer Vision and Pattern Recognition. 7008-7024.

[41] Tim Salimans, Ian Goodfellow, Wojciech Zaremba, Vicki Cheung, Alec Radford, Xi Chen, and Xi Chen. 2016. Improved Techniques for Training GANs. In Advances in Neural Information Processing Systems, Vol. 29.

[42] Christian Szegedy, Vincent Vanhoucke, Sergey Ioffe, Jon Shlens, and Zbigniew Wojna. 2016. Rethinking the inception architecture for computer vision. In Proceedings of the IEEE conference on computer vision and pattern recognition. 2818-2826.

[43] Hao Tan and Mohit Bansal. 2019. Lxmert: Learning cross-modality encoder representations from transformers. In EMNLP.

[44] Aäron van den Oord, Nal Kalchbrenner, Lasse Espeholt, Koray Kavukcuoglu, Oriol Vinyals, and Alex Graves. 2016. Conditional Image Generation with PixelCNN Decoders. In NIPS.

[45] Ashish Vaswani, Noam Shazeer, Niki Parmar, Jakob Uszkoreit, Llion Jones, Aidan N Gomez, Łukasz Kaiser, and Illia Polosukhin. 2017. Attention is all you need. In Advances in neural information processing systems. 5998-6008.

[46] Ramakrishna Vedantam, C Lawrence Zitnick, and Devi Parikh. 2015. Cider: Consensus-based image description evaluation. In Proceedings of the IEEE conference on computer vision and pattern recognition. 4566-4575.

[47] Tao Xu, Pengchuan Zhang, Qiuyuan Huang, Han Zhang, Zhe Gan, Xiaolei Huang, and Xiaodong He. 2018. Attngan: Fine-grained text to image generation with attentional generative adversarial networks. In Proceedings of the IEEE conference on computer vision and pattern recognition. 1316-1324.

[48] Xu Yang, Kaihua Tang, Hanwang Zhang, and Jianfei Cai. 2019. Auto-encoding scene graphs for image captioning. In Proceedings of the IEEE Conference on Computer Vision and Pattern Recognition. 10685-10694.

[49] Xu Yang, Hanwang Zhang, and Jianfei Cai. 2019. Learning to collocate neural modules for image captioning. In Proceedings of the IEEE International Conference on Computer Vision. 4250-4260. 
[50] Ting Yao, Yingwei Pan, Yehao Li, and Tao Mei. 2018. Exploring visual relationship for image captioning. In Proceedings of the European conference on computer vision (ECCV). 684-699.

[51] Han Zhang, Tao Xu, Hongsheng Li, Shaoting Zhang, Xiaogang Wang, Xiaolei Huang, and Dimitris N Metaxas. 2017. Stackgan: Text to photo-realistic image synthesis with stacked generative adversarial networks. In Proceedings of the IEEE international conference on computer vision. 5907-5915
[52] Luowei Zhou, Hamid Palangi, Lei Zhang, Houdong Hu, Jason Corso, and Jianfeng Gao. 2020. Unified vision-language pre-training for image captioning and vqa. In Proceedings of the AAAI Conference on Artificial Intelligence, Vol. 34. 13041-13049.

[53] Minfeng Zhu, Pingbo Pan, Wei Chen, and Yi Yang. 2019. Dm-gan: Dynamic memory generative adversarial networks for text-to-image synthesis. In Proceedings of the IEEE/CVF Conference on Computer Vision and Pattern Recognition. 